\title{
Cathepsin L expression in the carotid arteries of atherosclerotic swine
}

\author{
Ayisha Zaka Bashir ${ }^{1}$, Khalid Bashir ${ }^{1}$, William J. Hunter ${ }^{1}$, Devendra K. Agrawal ${ }^{2}$
}

\author{
'Department of Clinical Translational Sciences, School of Medicine, Creighton \\ University, Omaha. Nebraska, USA \\ ${ }^{2}$ Department of Translational Research, Western University of Health Sciences, \\ California, USA
}

Submitted: 11 September 2019

Accepted: 5 November 2019

Arch Med Sci Atheroscler Dis 2019; 4: e264-e267

DOI: https://doi.org/10.5114/amsad.2019.90153

Copyright @ 2019 Termedia \& Banach

Atherosclerosis is an inflammatory condition in which fatty deposits can clog the arteries and the buildups are called plaque [1]. Stroke remains a massive public health problem with tremendous health care costs. At least $20 \%$ of ischemic strokes are caused by carotid artery atherosclerotic plaque [2]. Even though there are phenomenal gains in clinical management of patients with symptomatic carotid artery disease, the molecular mechanisms and pathways leading to plaque instability remain poorly established $[3,4]$. Identification of the molecular markers of plaque instability along with signaling mechanisms may help in providing alternatives to surgical treatment and prevention of stroke. Cathepsin L (CTSL) is involved in inflammation and degradation of the extracellular matrix in the fibrous cap, causing destabilization of the plaque. These proteases serve as potential markers for plaque inflammation and vulnerability [5, 6]. At present it is still unclear how CTSL plays a role in the development of atherosclerotic plaque instability as well as plaque rupture and necrotic core formation $[7,8]$.

A pilot study was conducted on human carotid endarterectomy tissues collected anonymously. Plaques were marked as clinically asymptomatic (A) and symptomatic (S) male and female patients, aged between 50 and 75 years. The protein expression of CTSL in S (unstable) plaques compared to A (stable) plaques were analyzed by double immunofluorescence and fibrous cap and necrotic core were assessed by morphometric analysis. Our initial findings show increased expression of CTSL in symptomatic plaques. The increased expression of CTSL in S plaques highlights the potential role of CTSL in plaque instability and needs further investigation in animal models.

The porcine model is the most used and accepted model for cardiovascular diseases [9]. The same operative and imaging techniques used clinically in humans can be performed in swine [10, 11]. Previous studies conducted in swine show morphology and physiology of the lesions in the carotid arteries comparable to humans $[12,13]$. The swine model of atherosclerosis is documented as an appropriate model to investigate the pathophysiologic mechanisms leading to carotid artery atherosclerosis $[14,15]$. A pilot study was conducted by us on the swine model, to further investigate the role of CTSL in the pathogenesis of carotid atherosclerosis and to enhance knowledge of the mechanistic factors and key molecules in the development of unstable plaques in vivo. To our knowledge no such study has been conducted before.

\author{
Corresponding author: \\ Ayisha Zaka Bashir MBBS, \\ MS \\ School of Medicine \\ Creighton University \\ 2500 California Plaza \\ NE 68178,Omaha USA \\ Phone: 402-740-7394 \\ E-mail: ayishabashir@ \\ creighton.edu
}




\section{Material and methods}

The Institutional Animal Care and Use Committee of Creighton University approved the research protocol (IACUC\#1017) and the Yucatan micro swine were housed and maintained in the Animal Resource Facility of Creighton University, following the rules and regulations of $\mathrm{NIH}$ and USDA. The animals were divided into two groups. Each group had a total of four pigs each, depending on the intervention. Group one animals underwent angioplasty only, while group two animals underwent angioplasty and an injection of lipopolysaccharide (LPS) $100 \mu \mathrm{g}$ in normal saline locally in the carotid artery (once only) to induce further atherosclerosis. All animals were fed a high-cholesterol diet, purchased from Research Diets Inc, for 4-6 months.

The animals were given intramuscular anesthesia before the procedure, then started on isoflurane inhalation using a face mask followed by intubation with an endotracheal tube. After scrubbing and sterile draping, access to the vascular system was obtained after femoral cut down and exposure of the femoral artery. Once the femoral artery was punctured, the $6 \mathrm{Fr}$ sheath was introduced to keep the artery open. An angiographic guiding catheter $(6 \mathrm{Fr} A R 2 / 100 \mathrm{~cm})$ was pushed all the way to the carotid arteries on a guiding wire. Fluoroscopic evaluation was performed by administering non-ionic contrast media into the carotid arteries for fluoroscopic evaluation using a C arm (OEC 9900 Elite Vas 8, GE Healthcare). A clinical "coronary-type" angioplasty catheter with a balloon size of $2.5 \mathrm{~mm} \times 15 \mathrm{~mm}$ was gently pushed on the guide wire, until its deflated balloon was inside the left circumflex (LCX) coronary artery, single complete balloon inflation to 10 $15 \mathrm{~atm}$ pressure, depending on the vessel diameter using the inflation device with a pressure gauge (BasixTouch), then the balloon was deflated, and the patency of the artery was checked by performing angiography. Once the procedure was completed, the catheters were removed, the femoral artery sutured with prolene, and the leg incision closed with vicryl. After the procedure, the animal recovered under a thermal blanket and intravenous fluids continued until the animal was able to stand unassisted. Low-dose intramuscular buprenorphine was given to alleviate postoperative pain and repeated every 6-12 h. For the first 10 post-operative days, the animals were monitored daily for signs of pain and discomfort.

At 6 months after carotid intervention, angiogram and optical coherence tomography (OCT) imaging was performed using the C7-XR OCT intravascular imaging system (St. Jude Medical, St. Paul, MN). A high dose of barbiturates (Beuthanasia-D, $0.1 \mathrm{ml} / \mathrm{lb}$, i.v.) was administered to euthanize the animals. Swine carotids was removed quickly after euthanasia and fixed in $10 \%$ formalin buffer for $24 \mathrm{~h}$. The tissues were handled in a Sakura Tissue Tek VIP Tissue Processor and embedded in paraffin. Sections were cut at a thickness of $5 \mu \mathrm{m}$ using a microtome (Leica, Germany) and subsequently placed on slides for immunofluorescence evaluation.

\section{Histological preparation}

Histologic sections were cut at $6 \mu \mathrm{m}$, mounted on charged slides, and stained with hematoxylin-eosin (H\&E), trichome and Movat's pentachrome stain. Thickness was observed in an Olympus Slide Scanner microscope (Olympus VS120), using Image-pro software for analysis. The intimal changes and plaque thickness of the tissues were measured and analyzed using Student's $t$-test through SPSS statistical software.

\section{Immunofluorescence}

Tissue sections on the slides were deparaffinized in xylene, rehydrated in ethanol, washed in double-distilled water and treated with Lab Vision HIER Buffer L pH 9.0 (Thermo Fisher Scientific Inc.) at $90^{\circ} \mathrm{C}$ for $20 \mathrm{~min}$. The slides were then washed three times for $3 \mathrm{~min}$ each using $1 \mathrm{x}$ phosphate buffered saline (PBS) and then were blocked in normal horse serum from Vector Laboratories for $2 \mathrm{~h}$ at room temperature.

Immunohistochemistry was carried out using rabbit anti-pig CTSL (Sino biological), mouse anti-pig Cystatin C (Novus Biological), anti-TGF-B1 (Novus Biological), an anti-CD68 antibody (Santa Cruz Biotechnology) and $\alpha$-actin (abcam). The tissue sections were incubated with either mouse monoclonal anti-CD68, anti- $\alpha$ smooth muscle actin (CD 68 for identification of macrophages and $\alpha$-actin for smooth muscle cell identification), anti-CTSL antibody alone or in combination to examine the colocalization of CD68 and CTSL immunopositivity. Sections were also incubated with primary antibody anti-TGF- $\beta 1$, anti- $\alpha$ smooth muscle actin, anti-CD68, matching secondary antibodies 594 (red) or green 488 (anti- $\alpha$ smooth muscle actin, anti-CD68). The tissue sections were incubated with cystatin c alone, or double stained with CTSL and TGF-B1 antibodies. Labeled specimens were examined using an Olympus VS120. After washing with PBS, the slides were stained with DAPI, (4,6-diamidino-2-phenylindole) and the immunofluorescence was observed in the Olympus fluorescent microscope. DAPI, FITC (green) and TRITC (red) filters were used. The average fluorescence intensity was quantified in the samples using Image-Pro software and OlyVia 2.9 Desktop software. 


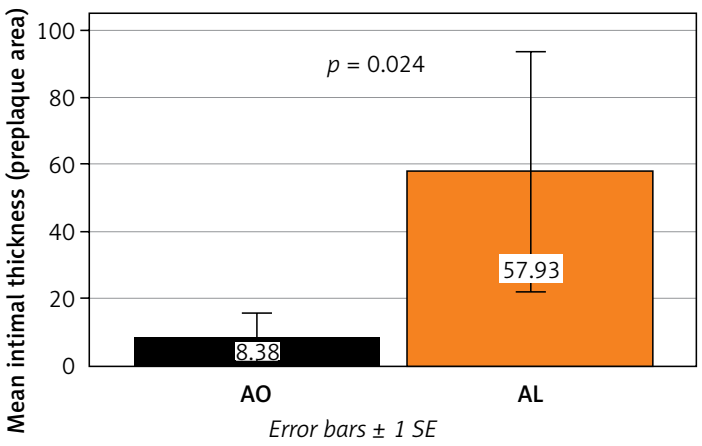

Figure 1. Bar graph measuring intimal thickness between the two groups

\section{Cell culture from carotid SMC}

Smooth muscle cells (SMCs) were prepared from carotid plaques by an established method developed by the CTS department. After gently scraping endothelial and adventitial layers, the medial layer was homogenized, washed in serumfree DMEM (Gibco BRL, Grand island, NY) and digested with $0.025 \%$ trypsin for $30 \mathrm{~min}$ at $37^{\circ} \mathrm{C}$ followed by $0.1 \%$ collagenase (Sigma, St. Louis, MO) digestion. The pellet was suspended in smooth muscle cell medium (ScienCell, Carlsbad, CA) and seeded on to $25 \mathrm{~cm}^{2}$ culture flasks and maintained at $37^{\circ} \mathrm{C}$ and $5 \% \mathrm{CO}_{2}$. The cells from the second to fifth passages were used. The phenotype and the homogeneity of isolated SMCs were confirmed by positive staining for smooth muscle $\alpha$-actin and caldesmon $[10,11]$. Once the cells were confluent, they were treated with CTSL, TGFB1 and cystatin C overnight.

\section{Statistical analysis}

Differences between the two plaque groups were analyzed by Student's $t$-test. Data were ex- pressed as percentage or mean \pm SD. Statistical differences between the two groups were calculated. All calculations were performed using IBM SPSS version 26.0 software. A probability value of $p<0.05$ was considered statistically significant.

\section{Results}

\section{Morphologic characteristics of carotid} arteries

The morphometric analysis in the pilot animal study shows greater intimal thickness and plaque formation in the angioplasty and LPS group (AL), as compared to the angioplasty only (AO) group, with a statistically significant $=0.024$ (Figure 1). The data are expressed in micrometers: $\mathrm{AL}=58.93 \pm 36, \mathrm{AO}=8.4 \pm 7.4, p=0.024$

Dual immunofluorescence was used to study co-localization of CTSL and a-actin SMA in carotid plaques. Antibodies to CTSL and anti- $\alpha$ smooth muscle actin $(\alpha-S M A)$, a marker for SMCs, were used to demonstrate the expression of CTSL in carotid arteries. It was observed that the CTSL immunofluorescence was greater in AL as compared to the angioplasty only group (Figures $2 \mathrm{~A}, \mathrm{~B}$ ). There is higher expression of CTSL and colocalization of both actin and CTSL in the AL group as compared to the $\mathrm{AO}$ group. The results were statistically significant, as shown by the values: $\mathrm{AL}=19.75 \pm 4.1$, $\mathrm{AO}=8.74 \pm 6.96, p=0.043$.

\section{Discussion}

The imbalance in the expression between CTSL and their inhibitor Cyst $C$, along with TGF- $\beta 1$, can trigger proteolysis of the extracellular matrix, leading to the pathogenesis of carotid artery disease and atherosclerosis. Monitoring circulating levels of CTSL and their endogenous inhibitor Cyst C may
A
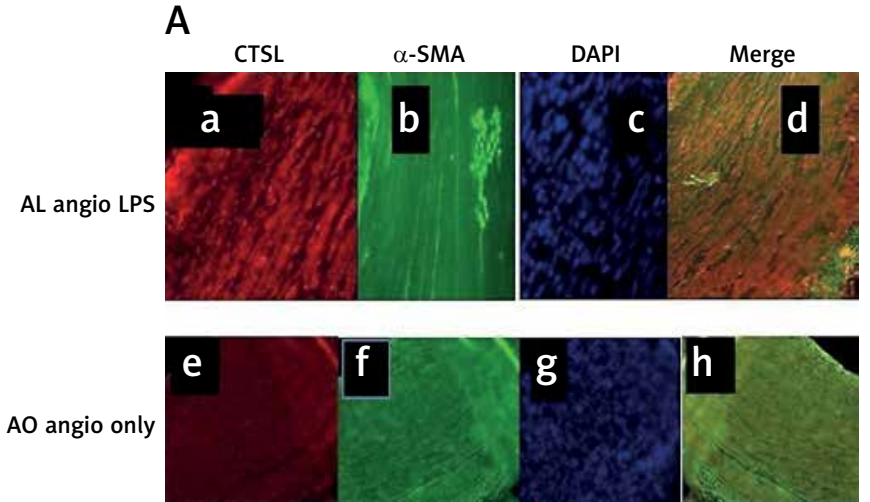

B

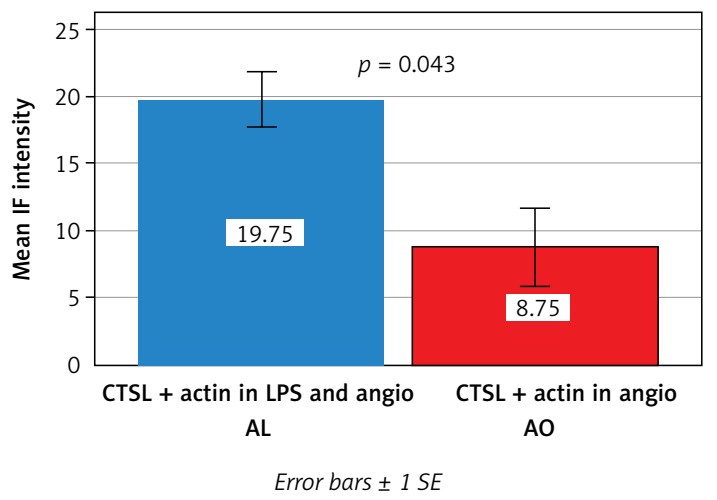

Figure 2. A-CTSL and $\alpha$-actin IF expression in both groups. Representative immunofluorescence images of Cathepsin L (CTSL) (red) $\alpha$-smooth muscle actin ( $\alpha$-SMA) (green) expression as visualized by dual immunofluorescence. Panels $\boldsymbol{a}, \boldsymbol{e}$-CTSL (red), $\boldsymbol{b}, \boldsymbol{f}$ - actin ( $\alpha$-SMA) (green), $\boldsymbol{c}, \boldsymbol{g}$ - nuclei labeled with DAPI, and $\boldsymbol{d}, \boldsymbol{h}$ merged immunopositivity to both CTSL and $\alpha$-SMA B - Graphical representation of mean IF intensity 
be considered useful as a biomarker and indicator of carotid artery stenosis $[14,15]$. CTSL interaction with cystatin- $C$, TGF- $\beta 1$ and carotid artery plaques may provide novel therapy for plaque stabilization and requires validation through further studies in animal models. Our pilot study demonstrates how a large-animal model is helpful in investigating the pathophysiologic process of plaques, but confirmation is needed in a small-animal model or in an in vitro study. Whether the higher expression of cathepsins in atherosclerotic plaques could contribute to the exaggerated intimal hyperplasia response seen in swine carotid arteries and models of atherosclerosis is another interesting possibility to explore in future studies $[12,13]$.

In conclusion, in this study the morphometric analysis of the swine carotid tissues showed greater intimal thickness and plaque formation in the angioplasty and LPS group (AL), as compared to the angioplasty only (AO) group, and the difference was statistically significant. The protein expression of CTSL is seen more in the AL carotid tissues, as shown by our experiments.

\section{Acknowledgments}

The research was made possible because of the valuable insight and guidance of Dr. Yiannis S. Chatzizisis (University of Nebraska Medical Center) and the Faculty and Staff of the Clinical Translational Science Department, School of Medicine, Creighton University, Omaha, Nebraska.

This work was supported by research grant R01HL144125 to DK Agrawal from the NHLBI-NIH, USA.

\section{Conflict of interest}

The authors declare no conflict of interest.

\section{References}

1. Skagen K, Skjelland M, Zamani M, Russell D. Unstable carotid artery plaque: new insights and controversies in diagnostics and treatment. Croat Med J 2016; 57: 311-20.

2. Benjamin EJ, Blaha MJ, Chiuve SE, et al.; the American Heart Association Statistics Committee and Stroke Statistics Subcommittee. Heart disease and stroke statistics - 2017 update: a report from the American Heart Association. Circulation 2017; 135: e229-445.

3. Li W, Kornmark L, Jonasson L, et al. Cathepsin L is significantly associated with apoptosis and plaque destabilization in human atherosclerosis. Atherosclerosis 2009; 202: 92-102.

4. Abd-Elrahman I, Meir K, Kosuge H, et al. Characterizing cathepsin activity and macrophage subtypes in excised human carotid plaques. Stroke 2016; 47: 1101-8.

5. Narula J, Garg P, Achenbach S, et al. Arithmetic of vulnerable plaques for noninvasive imaging. Nat Rev Cardiol 2008; 5: S2.

6. Kitamoto S, Sukhova GK, Sun J, et al. Cathepsin L deficiency reduces diet-induced atherosclerosis in low-den- sity lipoprotein receptor-knockout mice. Circulation 2007; 115: 2065-75.

7. Miyoshi N, Horiuchi M, Inokuchi Y, et al. Novel microminipig model of atherosclerosis by high fat and high cholesterol diet, established in Japan. In vivo 2010; 24 : 671-80.

8. Tellez A, Schuster DS, Alviar C, et al. Intramural coronary lipid injection induces atheromatous lesions expressing proinflammatory chemokines: implications for the development of a porcine model of atherosclerosis. Cardiovasc Revasc Med 2011; 12: 304-11.

9. Koskinas KC, Chatzizisis YS, Papafaklis MI, et al. Synergistic effect of local endothelial shear stress and systemic hypercholesterolemia on coronary atherosclerotic plaque progression and composition in pigs. Int J Cardiol 2013; 169: 394-401.

10. Gunasekar P, Swier VJ, Fleegel JP, et al. Vitamin D and macrophage polarization in epicardial adipose tissue of atherosclerotic swine. PLoS One 2018; 13: e0199411.

11. Rao VH, Rai V, Stoupa S, Subramanian S, et al. Tumor necrosis factor-alpha regulates triggering receptor expressed on myeloid cells-1-dependent matrix metalloproteinases in the carotid plaques of symptomatic patients with carotid stenosis. Atherosclerosis 2016; 248: 160-9.

12. Lee SG, Oh J, Bong SK, et al. Macrophage polarization and acceleration of atherosclerotic plaques in a swine model. PLoS One 2018; 13: e0193005.

13. Cai J, Zhong H, Wu J, et al. Cathepsin L promotes vascular intimal hyperplasia after arterial injury. Mol Med 2017; 23: 92.

14. Cipollone F, Fazia M, Mincione $G$, et al. Increased expression of transforming growth factor-beta 1 as a stabilizing factor in human atherosclerotic plaques. Stroke 2004; 35: 2253-7.

15. Liu J, Sukhova GK, Sun JS, et al. Lysosomal cysteine proteases in atherosclerosis. Arterioscler Thromb Vasc Biol 2004; 24: 1359-66. 